\title{
Ultraviolet modifications of dispersion relations in effective field theory
}

\author{
Robert C. Myers ${ }^{1,2}$ and Maxim Pospelov ${ }^{3,4}$ \\ 1 Perimeter Institute for Theoretical Physics, 35 King Street North, Waterloo, Ontario N2J 2W9, Canada \\ 2 Department of Physics, McGill University, Montréal, Québec H3A 2T8, Canada \\ ${ }^{3}$ Department of Physics and Astronomy, University of Victoria, Victoria, BC, V8P 1A1, Canada \\ ${ }^{4}$ Centre for Theoretical Physics, University of Sussex, Brighton BN1 9QJ, UK
}

\begin{abstract}
The existence of a fundamental ultraviolet scale, such as the Planck scale, may lead to modifications of the dispersion relations for particles at high energies, in some scenarios of quantum gravity. We apply effective field theory to this problem and identify dimension 5 operators that do not mix with dimensions 3 and 4 and lead to cubic modifications of dispersion relations for scalars, fermions, and vector particles. Further we show that, for electrons, photons and light quarks, clock comparison experiments bound these operators at $10^{-5} / M_{\mathrm{Pl}}$.
\end{abstract}

\section{INTRODUCTION}

The Planck mass, $M_{\mathrm{Pl}}$, a dimensional parameter determining the strength of gravitational interaction, remains a source of conceptual problems for quantum field theories. When the momentum transfer in a process of two particle collision is comparable to the Planck mass, the graviton exchange becomes strong, signifying a breakdown of the perturbative field theory description. Even without having a fully consistent fundamental theory at hand, one can hypothesize several broad categories of the low-energy effects induced by $M_{\mathrm{Pl}}$. The first group of such theories has only minor modifications due to the existence of new physics at $M_{\mathrm{Pl}}$. By minor modifications we understand that all "sacred" symmetries (Lorentz symmetry, CPT, spin-statistics, etc.) of the field theory remain unbroken at low energies. String theory in the critical dimension and in the simplest background reduces to field theory and gravity at the scales lower than $M_{s}$, and provides a perfect example of this category. In this case the chances to probe $1 / M_{\mathrm{Pl}}$ suppressed effects are very remote, as one has to have a unrealistically large momentum transfers. Consequently, the propagation of a free particle with large energy/momentum is immune to the effects of new physics, as all corrections to the dispersion relation could be cast in the form of $\left(p^{2}\right)\left(p^{2 n} / M_{\mathrm{Pl}}^{2 n}\right)=\left(m^{2 n+2} / M_{\mathrm{Pl}}^{2 n}\right)$ where $m$ is the mass of the particle and $p$ is the four-momentum.

In the second class of scenarios, that include loop quantum gravity, the $M_{\mathrm{Pl}}$ effects have much more "vivid" properties. In this approach, the discrete nature of space at short distances is expected to induce violations of Lorentz invariance and CPT. Such violations are also often discussed in a broader context using field theoretical language [1]. Here one might assume a perfectly Lorentz symmetric, CPT conserving action at $1 / M_{\mathrm{Pl}}^{0}$ order, and account for the existence of the Lorentz breaking terms via a set of higher dimension operators. This would lead to modifications of the dispersion relation for a free particle as terms of the form $E^{n+2} / M_{\mathrm{Pl}}^{n}$ can appear. Such effects can be searched for with astrophysical tools [2-5] or with high precision low-energy experiments $[6,7]$.
In this Letter, we consider cubic modifications of dispersion relations, which would appear at the leading order $1 / M_{\mathrm{Pl}}$ and which have received considerable attention in the literature recently [2-5]. In the language of the effective field theory, such modifications can be described by the dimension 5 operators. Although dimension 3,4 operators were extensively studied [1], dimension 5 operators remain unclassified and poorly explored. Lorentz breaking can be achieved by the introduction of an "external" background with open Lorentz indices. While more complicated choices are conceivable, we consider the case where a preferred frame is defined by a fixed time-like four-vector $n^{a}$. Within this framework, we study cubic modifications of the dispersion relation for scalars, vectors and fermions. Specializing the operators to Standard Model particles, we show that the cubic modification is not possible for the Higgs particles, must have an opposite sign for opposite chiralities of photons, and is independent for different chiralities of fermions. Note that the commonly studied dispersion relations [2-4] do not conform with those derived here.

Further we consider certain experimental bounds on these operators. It is important to realize that the introduction of any preferred frame leads to spatial anisotropy. The four-vector $n^{a}$ that characterizes the preferred frame may have a dominant component $n^{0}$ in some cosmic frame, but the motion of our galaxy, Solar system and Earth will create spatial components $n_{i} \sim 10^{-3}$ for a terrestrial observer [8]. Hence clock comparison experiments [9] or experiments with spin-polarized matter [10] searching for the spatial anisotropy can impose stringent bounds on violations of Lorentz symmetry in this context. Recently direction-sensitive clock comparison results were used to constrain the dispersion relation for nucleons [7]. Here with a direct application of the spin precession bounds, we derive $O(1)$ limits for leptons, $O\left(10^{-5}\right)$ for photons and $O\left(10^{-8}\right)$ for quarks in units of $1 / M_{\mathrm{Pl}}$. The results in the lepton sector can be improved to the level of $10^{-5} M_{\mathrm{Pl}}^{-1}$ with a renormalization group analysis. Comparing to recent calculations [11-13], we find that these bounds already present an interesting challenge for loop quantum gravity. 


\section{CUBIC MODIFICATIONS OF DISPERSION RELATIONS BY DIMENSION 5 OPERATORS}

In the framework of low energy effective field theory, the modified dispersion relations should be derived from some appropriate modification of the kinetic terms in the Lagrangian. In this paper we shall assume that shortdistance physics does not generate dimension three and four terms directly. Even though this assumption has serious theoretical problems (e.g., one wants a symmetry to forbid such operators), it is quite safe from the practical side, because even if these terms exist they are already severely constrained by experiment (see, e.g., [1]).

At the next level are dimension five operators which would lead to $O\left(E^{3}\right)$ modifications of the dispersion relations. We adopt the simplest approach where Lorentz symmetry is broken a background four vector $n^{a}$ (with $n \cdot n=1)$. We construct operators satisfying six generic criteria:

1. Quadratic in the same field

2. One more derivative than the usual kinetic term

3. Gauge invariant

4. Lorentz invariant, except for the appearance of $n^{a}$

5. Not reducible to lower dimension operators by the equations of motion

6. Not reducible to a total derivative

Conditions 2 and 5 ensure that these operators lead to $O\left(E^{3}\right)$ modifications of the dispersion relations, rather than $O\left(E^{2} m\right)$ or $O\left(E m^{2}\right)$, where $m$ is the mass of the particle. Our working assumption will also be that these operators are naturally suppressed by a factor of $1 / M_{\mathrm{Pl}}$, and that $m, E \ll M_{\mathrm{Pl}}$. This scaling ensures that all operators of dimension five can be regarded as small perturbations. We shall consider cases of a scalar, fermion and vector particle.

Scalar: We work with a complex scalar field with the standard dimension four Lagrangian: $\mathcal{L}_{0}=|\partial \Phi|^{2}-$ $m^{2}|\Phi|^{2}$. Hence the leading order equation of motion yields $\left(\square+m^{2}\right) \Phi=0$, or in momentum space with $\Phi \sim \exp (-i k \cdot x),\left(-k^{2}+m^{2}\right) \Phi(k)=0$.

It is relatively easy to see that there is only one possible term which appears at the dimension five level

$$
\mathcal{L}_{s}=i \frac{\kappa}{M_{\mathrm{Pl}}} \bar{\Phi}(n \cdot \partial)^{3} \Phi
$$

which is odd under $C P T$ and charge conjugation. The corresponding equation of motion becomes

$$
\left(\square+m^{2}\right) \Phi=i \frac{\kappa}{M_{\mathrm{Pl}}}(n \cdot \partial)^{3} \Phi .
$$

Another possible operator, $\bar{\Phi} n \cdot \partial \square \Phi$, is reducible to $m^{2} \bar{\Phi} n \cdot \partial \Phi$ and does not effect the dispersion relation in a significant way. In momentum space (with $n \cdot \partial \sim-i E$ ), and in the Lorentz frame where $n^{a}=(1,0,0,0)$, we have the dispersion relation

$$
E^{2} \simeq|\vec{p}|^{2}+m^{2}+\frac{\kappa}{M_{\mathrm{Pl}}}|\vec{p}|^{3}
$$

where we have used $E \simeq|\vec{p}|$ for high energies.

Note that for a real scalar (like the Standard Model Higgs), the operator $\mathcal{L}_{s}$ is the total derivative and does not produce a cubic modification of the dispersion relation. For the case of complex scalar, the introduction of $\mathcal{L}_{s}$ respects the phase invariance of $\mathcal{L}_{0}$. The dimension five operator breaking this symmetry, e.g., $\Phi(n \cdot \partial)^{3} \Phi$ vanishes again as a total derivative and is forbidden if the phase symmetry is gauged.

Vector: We work with a U(1) gauge field for which the leading kinetic term is $\mathcal{L}_{0}=-F^{2} / 4$. (Extending the following to a nonabelian vector is straightforward.) Hence the leading order equations of motion are just the Maxwell equations: $\partial_{a} F^{a b}=0$. After gauge fixing $\partial \cdot A=0$, this yields $\square A_{a}=0$, or in momentum space $k^{2} A_{a}(k)=0$. Again, we wish to modify the dispersion relation at $O\left(E^{3}\right)$ and so the new terms should satisfy the constraints listed above. Keeping in mind the leading order Maxwell equations in vacuum and the Bianchi identities $\partial_{[a} F_{b c]}=0$, one finds that there is only one term with the desired form and which produces a nontrivial modification of the dispersion relations

$$
\mathcal{L}_{\gamma}=\frac{\xi}{M_{\mathrm{Pl}}} n^{a} F_{a d} n \cdot \partial\left(n_{b} \tilde{F}^{b d}\right),
$$

where $\tilde{F}^{a b}=\frac{1}{2} \varepsilon^{a b c d} F_{c d}$. Note that this operator is odd under $C P T$ and even under charge conjugation. The equation of motion becomes

$$
\square A_{a}=-\frac{\xi}{M_{\mathrm{Pl}}} \varepsilon_{a b c d} n^{b}(n \cdot \partial)^{2} F^{c d}
$$

where we used Bianchi identity and $\partial \cdot A=0$. To identify the effect of the new term on the dispersion relation, we go to momentum space and select photons moving along the $z$ axis with $k^{a}=(E, 0,0, p)$. Then for transverse polarizations along the $x$ and $y$ axes,

$$
\left(E^{2}-p^{2} \pm \frac{2 \xi}{M_{\mathrm{Pl}}} p^{3}\right)\left(\epsilon_{x} \pm i \epsilon_{y}\right)=0
$$

where we have used $E \simeq p$ to leading order and chosen the "rest frame" for $n^{a}$ as before. Hence the sign of the cubic term is determined by the chirality (or circular polarization) of the photons. As in the case of optical activity, this leads to the rotation of the plane of polarization for linearly polarized photons, which is used to bound $\xi$ in [5]. Given that $\mathcal{L}_{\gamma}$ is unique, the common approach [2-4] of postulating a cubic dispersion relation which is chirality independent is incompatible with effective field theory.

Spinor: We work with a Dirac spinor for which the leading kinetic term is: $\mathcal{L}_{0}=\bar{\Psi}(i \not \partial-m) \Psi$. Again, we wish to modify the dispersion relation at $O\left(E^{3}\right)$ and so consider new terms satisfying the constraints listed above. Keeping in mind the leading order equations, one finds that there are only two terms with the desired form 


$$
\mathcal{L}_{f}=\frac{1}{M_{\mathrm{Pl}}} \bar{\Psi}\left(\eta_{1} \not h+\eta_{2} \not h \gamma_{5}\right)(n \cdot \partial)^{2} \Psi
$$

Both operators break CPT, with $\eta_{1}$ being charge conjugation odd and $\eta_{2}$ charge conjugation even. Now the equation of motion takes the form

$$
(i \not \partial-m) \Psi=-\frac{1}{M_{\mathrm{Pl}}}\left(\eta_{1} \not h+\eta_{2} \not h \gamma_{5}\right)(n \cdot \partial)^{2} \Psi
$$

To produce an expression that is readily identified with a dispersion relation, one operates on both sides with $(i \not \partial+m)$ to produce

$$
\left(\square+m^{2}\right) \Psi=\frac{2 i}{M_{\mathrm{Pl}}}\left(\eta_{1}+\eta_{2} \gamma_{5}\right)(n \cdot \partial)^{3} \Psi
$$

where we have again dropped terms of order $m / M_{\mathrm{Pl}}$. Hence the modified dispersion relation becomes

$$
\left(E^{2}-|\vec{p}|^{2}-m^{2}-\frac{2|\vec{p}|^{3}}{M_{\mathrm{Pl}}}\left(\eta_{1}+\eta_{2} \gamma_{5}\right)\right) \Psi=0
$$

with $E \simeq|\vec{p}|$ for high energies. At high energies (i.e., $E^{2} \gg m^{2}$ ), we can choose spinors as eigenspinors of the chirality operator and redefine coupling constants as $\eta_{R, L}=\eta_{1} \pm \eta_{2}$. In the extension of the Standard Model, the chiral choice for $\eta$ couplings is prescribed by gauge invariance. Previous studies [3,4] consider only chirality independent dispersion relations for fermions and so implicitly fix $\eta_{2}=0$.

Above we have identified interesting operators which modify the dispersion relations at cubic order. One could also consider frame-dependent modifications of the interaction terms between, e.g., photons and electrons. However, these would not effect the threshold tests which are usually studied, e.g., [3]. On the other hand, they may play a role in indirect tests as those considered below.

As a further technical aside, one can see a very serious potential problem with divergences at the loop level. For example, it is easy to show that when inserted in a self-energy loop, the $\eta_{2}$ operator generates a dimension 3 operator $\Lambda_{U V}^{2} M_{\mathrm{Pl}}^{-1} \bar{\psi} h \gamma_{5} \psi$ with $\Lambda_{U V}$, the ultra-violet cutoff on the momentum integral. The latter cannot be lower than the electroweak scale or SUSY breaking scale. However, even assuming such a low cutoff, the bounds of [1] on these dimension three operators restrict all the couplings, $\eta_{i}, \xi, \kappa$ to be smaller than $10^{-10}$. However, we may evade this bound as follows: The external tensor $n^{a} n^{b} n^{c}$ appearing in all of the operators above may be separated into a vector piece and a traceless symmetric tensor $C^{a b c}=n^{a} n^{b} n^{c}-\frac{1}{6}\left(n^{a} g^{b c}+\right.$ cyclic $)$. The quadratic divergences above are associated with the vector part only, while the operators proportional to $C^{a b c}$ can generate only logarithmic divergences which should be interpreted as the renormalization group evolution of these operators. Thus, to be consistent with the assumption about the absence of large dimension 3 or 4 operators, we make the substitution $n^{a} n^{b} n^{c} \rightarrow C^{a b c}$ in the following. Note that this change does not affect the dispersion relations in the regime $E \gg m$.

\section{LOW-ENERGY LIMITS ON DIMENSION FIVE OPERATORS}

Modifications of dispersion relations for stable particles such as electrons, light quarks, and photons could be searched for using the astrophysical probes [2-5]. Here we show that terrestrial limits imposed by the clock comparison experiments are equally and sometimes more sensitive to dimension 5 operators. Indirect limits exploit the idea that the choice of $n^{a}=(1,0,0,0)$ defines a preferred frame that does not coincide with the laboratory frame on the Earth [8]. Assuming that the rest frame for $n^{a}$ is related somehow to either the cosmic or galactic frame, the typical size of the spatial component of $n$ is $10^{-3}$.

Limits on the operators involving electrons and electron neutrinos are especially easy to derive. We use the fact that best tests of directional sensitivity in the precession of electrons limit the size of interaction between the external direction and the electron spin at the level of $10^{-28} \mathrm{GeV}$ [10]. This immediately translates into the following limit on the coefficients $\eta_{L}$ and $\eta_{R}$ that parametrize the effective interaction of the form (7) for left-handed leptons and right-handed electrons:

$$
\left|\eta_{L}^{e}-\eta_{R}^{e}\right| \lesssim \frac{10^{-28} \mathrm{GeV} M_{\mathrm{Pl}}}{m_{e}^{2}\left|n_{i}\right|} \simeq 4
$$

where we use $M_{\mathrm{Pl}} \equiv 10^{19} \mathrm{GeV}$. The square of the electron mass originates from $\left(n^{0} \partial_{t}\right)^{2}$ acting on the electron wave function. The orthogonal combination, $\eta_{L}^{e}+\eta_{R}^{e}$, is almost not constrained, as it does not contribute into the electron spin Hamiltonian. Similarly, we do not constrain operators involving second and third generation leptons. Note that our limit is comparable to the existing constraints [3]. However the latter analysis assumed $\eta_{2}=0$ and so our result is a complementary constraint.

The absence of a preferred direction is checked with even greater precision using nuclear spin, which translates into more stringent limits on new operators for the light quarks. The photon operator (4) will also contribute because of the electromagnetic interactions inside the nucleon. To use the best experimental limits of $10^{-31} \mathrm{GeV}$ on the coupling of $n_{i}$ to neutron spins [9], we must relate the photon and quark operators with nucleon spin. Introducing dimension five operators for left-handed doublet $\psi_{Q}$ and right-handed singlets $\psi_{u}$ and $\psi_{d}$, members of the first generation of quarks

$$
\mathcal{L}_{q}=\frac{C^{a b c}}{M_{\mathrm{Pl}}} \sum_{i=Q, u, d} \eta_{i} \bar{\psi}_{i} \gamma_{a} \partial_{b} \partial_{c} \psi_{i}
$$

at the nucleon level we have

$$
\begin{array}{r}
\eta_{1, N}=a_{u}\left(\eta_{u}+\eta_{Q}\right)+a_{d}\left(\eta_{d}+\eta_{Q}\right) \\
\eta_{2, N}=b_{u}\left(\eta_{u}-\eta_{Q}\right)+b_{d}\left(\eta_{d}-\eta_{Q}\right)+b_{\gamma} \xi
\end{array}
$$

where $\eta_{1(2), N}$ are the $\eta_{1}$ and $\eta_{2}$ couplings for nucleons defined in (7). Note that $\xi$ enters only in the $\eta_{2}$ coupling 
for nucleons because both are even under the charge conjugation. In (13) $a_{u, d}$ and $b_{u, d}$ are the matrix elements that could be obtained as the moments of the experimentally measured structure functions: $a_{d} \sim 0.4, a_{u} \sim 0.1$, $b_{d} \sim 0.1, b_{u} \sim-0.05$ for the neutron and charge inverted values for the proton. To relate the photon operator with nucleon, we use the simplest vector dominance model and obtain at one-loop level $b_{\gamma} \sim 0.13 \alpha /(4 \pi)$ for neutron and $b_{\gamma} \sim 0.24 \alpha /(4 \pi)$ for proton. This results in the limit:

$$
\left|\left(\eta_{d}-\eta_{Q}\right)-0.5\left(\eta_{u}-\eta_{Q}\right)+10^{-3} \xi\right| \lesssim 10^{-8}
$$

Barring accidental cancellations, one can place separate limits on $\eta_{u, d}-\eta_{Q}$ at $10^{-8}$ and on $\xi$ at $10^{-5}$ level. The orthogonal combinations $\eta_{u, d}+\eta_{Q}$ are less constrained because they enter only in the quadrupole coupling between the nuclear spin and external direction, and thus are suppressed by an additional factor of $\left|n_{i}\right| \sim 10^{-3}[7]$.

So far we have neglected the fact that the low energy values for the couplings $\eta_{i}$ and $\xi$ taken at the normalization scale of $1 \mathrm{GeV}$ do not coincide with the high-energy values for the same couplings generated at $M_{\mathrm{Pl}}$. With a simple one-loop analysis of the renormalization group equations, we find that several bounds can be strengthened. Leaving the details for elsewhere, our results are: $\left|\eta_{Q, u, d}\right|,|\xi| \lesssim 10^{-6}$ and $\left|\eta_{L, R}^{e}\right| \lesssim 10^{-5}$ with the same normalization on $1 / M_{\mathrm{Pl}}$. The constraints may also be improved by assuming a GUT scenario.

In summary, we have shown that effective field theory provides a framework where one can derive stringent bounds on Planck scale interactions from terrestrial experiments. The resulting limits enhance and generalize the bounds obtained previously in [7]. They are generally far more sensitive [7] than those previously derived by considering astrophysical phenomena [3] where the typical sensitivity is $O(1)$ in units of $M_{\mathrm{Pl}}^{-1}$. Actually the birefringence induced by $\mathcal{L}_{\gamma}$ can be used to bound $|\xi| \lesssim 10^{-4}$ with astronomical observations [5], but our bound improves on this result by roughly an order of magnitude. Recently [4] infers a striking bound $\eta_{L}^{e}+\eta_{R}^{e} \gtrsim-10^{-9}$ (assuming $\eta_{L}^{e}-\eta_{R}^{e}=0$ ) from the observation of synchrotron radiation from astrophysical sources. Here our results provide an upper bound for positive couplings.

Following [7], we compare our results to semi-classical calculations which have appeared using loop quantum gravity [11-13]. Examining the interactions induced for a Dirac fermion [12] shows that $\eta_{1}=0$ and that while $\eta_{2}$ is nonvanishing, it is suppressed an additional small parameter related to the coherence scale of the gravitational wave function. Hence these calculations seem to be in accord with the stringent bounds derived for the fermion operators. In contrast, the results of [11] suggest that $\xi$ should be $O(1)$, which stands in stark contradiction with the bound derived here. Hence our bounds seem to be in conflict with the present calculations [11-13]. However, the latter must be viewed as somewhat heuristic. So the immediate challenge for loop quantum gravity is to provide rigorous predictions that may be compared to experiment. Despite the many unresolved theoretical issues, it is truly remarkable that precision experiments can already confront quantum gravity calculations with concrete observational bounds.

We thank Giovanni Amelino-Camelia, Cliff Burgess, Ted Jacobson, Joe Lykken, Guy Moore, Subir Sarkar, Lee Smolin and especially Seth Major for useful conversations. This research is supported in part by NSERC of Canada, Fonds FCAR du Québec and PPARC UK.

[1] D. Colladay and V.A. Kostelecky, Phys. Rev. D55 (1997) 6760; S.R. Coleman and S.L. Glashow, Phys. Rev. D59 (1999) 116008; S.M. Carroll, G.B. Field and R. Jackiw, Phys. Rev. D41 (1990) 1231.

[2] G. Amelino-Camelia et al., Nature 393 (1998) 763; G. Amelino-Camelia and T. Piran, Phys. Rev. D64 (2001) 036005; G. Amelino-Camelia, Phys. Lett. B528 (2002) 181; S. Sarkar, Mod. Phys. Lett. A17 (2002) 1025.

[3] T. Jacobson, S. Liberati and D. Mattingly, Phys. Rev. D66 (2002) 081302; hep-ph/0209264; T.J. Konopka and S.A. Major, New J. Phys. 4 (2002) 57.

[4] T. Jacobson, S. Liberati and D. Mattingly, astro$\mathrm{ph} / 0212190$.

[5] R.J. Gleiser and C.N. Kozameh, Phys. Rev. D64 (2001) 083007.

[6] G. Amelino-Camelia, Nature 398 (1999) 216; I. Mocioiu, M. Pospelov and R. Roiban, Phys. Lett. B489 (2000) 390; G.Z. Adunas, E. Rodriguez-Milla and D.V. Ahluwalia, Phys. Lett. B485 (2000) 215; R. Bluhm, hep-ph/0111323.

[7] D. Sudarsky, L. Urrutia and H. Vucetich, Phys. Rev. Lett. 89 (2002) 231301.

[8] I.Mocioiu and M.Pospelov, Phys. Lett. B534 (2002) 114.

[9] T.E. Chupp et al., Phys. Rev. Lett. 72, 2363 (1994); R.E. Stoner et al., Phys. Rev. Lett. 77, 3971 (1996); D. Bear et al., Phys. Rev. A57, 5006 (1995); C.J. Berglund et al., Phys. Rev. Lett. 75, 1879 (1995); D. Bear, et al. Phys. Rev. Lett. 85 (2000) 5038 [Errat. 89 (2002) 209902].

[10] E.G. Adelberger et al., in Physics Beyond the Standard Model, 1998, Santa Fe (World Scientific, Singapore, 1999); M.G. Harris, PhD Thesis, University of Washington, 1998; B.R. Heckel et al., in Proceedings of the International Conference on Orbis Scientiae, 1999, Coral Gables (Kluwer, New York, 2000).

[11] R. Gambini and J. Pullin, Phys. Rev. D59 (1999) 124021; J. Alfaro, H.A. Morales-Tecotl and L.F. Urrutia, Phys. Rev. D65 (2002) 103509.

[12] J. Alfaro, H.A. Morales-Tecotl and L.F. Urrutia, hepth/0208192.

[13] H. Sahlmann and T. Thiemann, gr-qc/0207030; grqc/0207031. 